\title{
Mapping the electronic structure of polypyrrole with image-based electrochemical scanning tunnelling spectroscopy
} \author{
Pereira ${ }^{[a]}$ and Angel Cuesta ${ }^{[b],{ }^{*}}$ \\ [a] Dr. R. Gonçalves, MSc. R. S. Paiva and Prof. Dr. E. C. Pereira \\ Department of Chemistry \\ Federal University of São Carlos \\ Mail Box 676, CEP 13565-905, São Carlos - SP, Brazil \\ [b] Dr. R. Gonçalves, Dr. A. M. R. Ramírez, Dr. J. A; Mwanda and Prof. Dr. A. Cuesta \\ Department of Chemistry \\ School of Natural and Computing Sciences, University of Aberdeen \\ Aberdeen AB24 3UE, UK \\ E-mail: angel.cuestaciscar@abdn.ac.uk \\ [c] Dr. A. M. R. Ramírez \\ Department of Chemistry \\ Pontificia Universidad Católica de Chile \\ Av. Vicuña Mackenna 4860, 7820436-Macul, Santiago, Chile \\ Current address: Laboratorio de Electroquímica y Materiales Aplicados \\ Centro de Nanotecnología Aplicada, Facultad de Ciencias, Universidad Mayor \\ Av. Alemania 0281, 4801043-Temuco, Chil
}

Roger Gonçalves ${ }^{[a, b]}$, Robert S. Paiva ${ }^{[a]}$, Andrés M. R. Ramírez ${ }^{[b, c]}$, Jonathan A. Mwanda ${ }^{[b]}$, Ernesto C.

\begin{abstract}
Conducting polymers are semiconductors whose applications cover a wide range of devices. Their versatility is due, in addition to other factors, to properties that can be easily modulated according to the intended application. It is therefore important to study and map the electronic structure of these materials for a better correlation between structure and properties. Electrochemical scanning tunnelling spectroscopy (EC-STS) can be a powerful tool to characterize the electronic structure of the semiconductor interface. In this work we have used image-based EC-STS (IB-ECSTS) to describe quantitatively the band structure of an electrochemically deposited polypyrrole film. IB-EC-STS located the band edge of the polymer's valence band (VB) at $0.95 \mathrm{~V}$ vs. RHE ($5.33 \mathrm{eV}$ in the absolute potential scale) and the intragap polaron states formed when the polymer is doped, at $0.46 \mathrm{~V}$ vs. RHE (-4.84 $\mathrm{eV}$ ). The IB-EC-STS data were cross checked with electrochemical impedance spectroscopy (EIS) and Mott-Schottky analysis of the interfacial capacitance. The DOS spectrum obtained from EIS data is consistent with the STS-deduced location of the VB and the polarons.
\end{abstract}

\section{Introduction}

Understanding the electronic structure of conducting polymers (CPs) is necessary for a detailed understanding of the behaviour of these materials, whose mechanism of electronic conduction is different from that characteristic of inorganic semiconductors. It is therefore important to acquire the experimental information required to build a complete model of their band structure, which governs their properties. ${ }^{[1,2]}$

Due to the size of CP molecules, atomic orbitals in the polymer chains combine to give rise to bands that extend throughout the entire macromolecule. ${ }^{[3]}$ As in the case of inorganic semiconductors, the conductivity of the undoped material is associated to either free electrons in the conduction band $(\mathrm{CB})$ or free holes in the valence band (VB) generated either by photo-excitation or (if the band gap is sufficiently small compared with $k T)$ by thermal excitation of electrons from the $V B$ into the $C B$. In the case of inorganic semiconductors, the conductivity can be increased by injecting electrons or holes in either the $\mathrm{CB}$ or the VB through $n$ or $p$ doping, respectively, which typically results in the creation of an associated point defect in the material. On the other hand, doping of CPs is due to oxidation or reduction processes in the polymer, and does not result in the injection of electrons or holes in the $\mathrm{CB}$ or the VB, respectively, but in the formation of solitons, polarons and, at high doping levels, bipolarons, localised within the energy gap. ${ }^{[4],[5]}$ In the particular case of $p$-doping a CP through oxidation, a radical cation (hole-polaron, spin 1/2) is created upon removal of an electron from the polymeric chain, ${ }^{[5]}$ which could be understood as a rearrangement of $\pi$ electrons that locally polarizes the polymer chain. In chemical terms, a polaron consists of a radical ion with unitary charge and a well-defined energy within the gap that can be detected, e.g., via optical spectroscopy ${ }^{[6]}$ (please note, however, that optical spectroscopy will provide the energy difference between the intragap level and the band edge, but never the exact location of the intragap level in a well-defined energy scale, unless the location of the band edge itself is also known). If a second electron is removed from the polymer chain, two situations can occur: (i) another holepolaron state is created, or (ii) if the polarons are close enough (i. e., at high doping levels ${ }^{[5,7]}$ ) they collapse in a single, doubly charged defect, a bipolaron (spin 0). ${ }^{\left[{ }^{-10]}\right.}$ In summary, like inorganic semiconductors, CPs can be doped in order to control their conductivity. ${ }^{[11]}$ However, the charge carriers are not electrons or holes inside the $C B$ and VB, respectively, but polarons and bipolarons located within the bandgap. ${ }^{12]}$

Polypyrrole (PPy) is one of the most studied CPs because of its low oxidation potential, the water solubility of the monomer and its low cost. ${ }^{[13]}$ These unique properties make it compatible with devices working in aqueous media, in applications like drug delivery, biosensors, artificial muscles and optoelectronic devices. ${ }^{[14]}$ PPy was the first CP to be characterized by its optoelectronic properties, which can be altered by doping/dedoping. ${ }^{[15]}$

Scanning Tunnelling Microscopy (STM) is a powerful technique based on the quantum tunnelling effect that allows obtaining images of surfaces with atomic resolution. ${ }^{[16]}$ When a sharp metallic tip is placed very close to a sample surface and a small bias voltage is applied, a tunnelling current flows through 
the gap between them. This tunnelling current can also flow through a liquid electrolyte, and it is measurable if the tip is properly isolated to decrease the magnitude of any faradaic currents below the level of the target tunnelling current, enabling atomic resolution images of electrode surfaces. ${ }^{[17,18]}$ The tunnelling current also depends strongly on the density of states, and tunnelling current $v s$. bias plots obtained during a fast scan (typically in the $\mathrm{V} \mathrm{s}^{-1}$ region) of the tip-sample bias (i.e., scanning tunnelling spectroscopy, STS) have been used to elucidate the band structure of conducting polymers in ultra-high vacuum $(\mathrm{UHV}) .{ }^{[19]} \mathrm{A}$ problem with this approach, however, is that the energy levels obtained by UHV-based STS cannot be related to any useful energy scale, like the reversible hydrogen electrode (RHE) or the absolute potential scale. This could be solved by doing STS at the electrode-electrolyte interface (electrochemical STS, EC-STS), where the potential of both tip and sample can be referred to a common reference electrode, but this is very challenging, because, at the high scan rates needed to scan the tip potential, faradaic and capacitive currents (which scale with the square root of and linearly with, respectively, the scan rate) easily obscure the tunnelling current. A way around this problem is to use imaged-based EC-STS (IB-EC-STS), in which the tip potential versus a suitable reference electrode is scanned during imaging in either positive or negative direction until the STM image disappears, which must coincide with the moment at which a band edge is crossed and the Fermi level of the tip falls within the sample's band gap (where neither tunnelling from the tip to the full VB nor from the empty $C B$ to the tip is possible). This approach was used by Sanz and co-workers ${ }^{[20]}$ to elucidate the band structure of the passive film on an iron electrode, and that work remains, to the best of our knowledge, the only example of IB-EC-STS.

In this work, IB-EC-TS and energy-resolved electrochemical impedance spectroscopy (EIS) were combined to map the electronic structure of a PPy film and to obtain a quantitative description of its electronic structure.

\section{Results and Discussion}

\section{Electrochemical characterization}

Figure 1a shows the cyclic voltammograms recorded during the growth of a PPy film on a Pt(111) electrode in a perchloric acid solution containing pyrrole. During growth, the current in the double-layer region increases linearly with increasing number of cycles (Fig. 1a, inset), as expected. After the $20^{\text {th }}$ growth cycle, the voltammetric profile of the PPy film in a pyrrole-free perchloric acid solution agrees with those described in previous work for PPy in acidic media (Figure 1b). ${ }^{[21-23]}$ The cyclic voltammogram is characterised by an oxidation process that starts around $0.12 \mathrm{~V}$ and peaks around $0.49 \mathrm{~V}$, corresponding to the $p$-doping of the polymer. The PPy oxidation-reduction process is very reversible, showing a coulombic efficiency (ratio between the oxidation and reduction charges) of $93.6 \%$.

Electrochemical impedance spectroscopy was used to obtain information about the electrochemical and electronic properties of the PPy film. The impedance results were fitted using a transmission line mode ${ }^{[21,24-28]}$, which is presented in the Supplementary Information (Part 2).
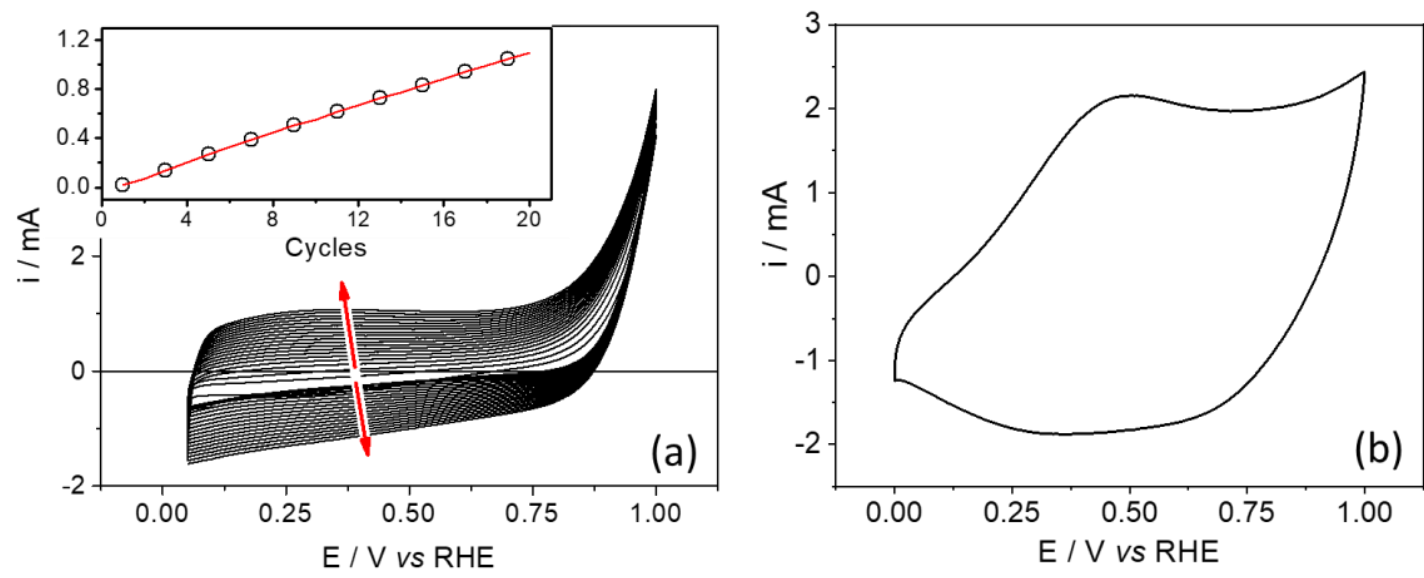

Figure 1. (a) Cyclic voltammograms recorded during the growth of polypyrrole from $0.1 \mathrm{~mol} \mathrm{~L}^{-1} \mathrm{HClO}_{4}+0.1$ mol $\mathrm{L}^{-1}$ pyrrole solutions on a Pt(111) electrode. The inset shows the linear increase of the current at $0.04 \mathrm{~V}$ with increasing number of cycles; (b) cyclic voltammetry of $\mathrm{PPy}$ on $\mathrm{Pt}(111)$ in pyrrole-free $0.1 \mathrm{M} \mathrm{HClO} 4 \mathrm{~mol}$ $\mathrm{L}^{-1}$. Scan rate: $0.05 \mathrm{~V} \mathrm{~s}^{-1}$.

A plot of the inverse of the squared capacitance (Figure 2a), as obtained from the impedance measurements, can be used to determine the apparent charge-carrier density and the apparent flat-band potential by fitting the data to the Mott-Schottky equation:

$$
\frac{1}{C^{2}}=\frac{2}{\varepsilon_{r} \varepsilon_{0} e N_{h}}\left(E_{\mathrm{fb}}-E+\frac{k_{\mathrm{B}} T}{e}\right)
$$

Equation 1

Where $\varepsilon_{\mathrm{r}}$ is the material's dielectric constant, $\varepsilon_{0}$ is the vacuum permittivity, $e$ is the elementary charge, $N_{h}$, is the density of majority charge carriers (in the PPy case, polaron and, at high doping levels, bipolaron holes), $E_{\mathrm{fb}}$ is the flat-band potential, $E$ is the applied potential, $k_{B}$ is the Boltzmann constant and $T$ is the 
temperature in Kelvin. Figure 2a shows such a plot and fit, from where an apparent flat-band potential of $0.11 \mathrm{~V}$ and an apparent hole polaron density of $3.31 \times 10^{19} \mathrm{~cm}^{-3}$ is obtained. We will discuss these values later, in the light of the information obtained from the impedance-derived distribution of density of states (DOS) and the IB-EC-STS data.

The potential dependent charge transfer resistance $\left(R_{\mathrm{ct}}\right)$, as obtained from EIS, can be used to calculate the DOS spectrum

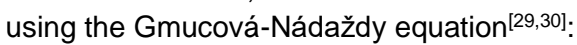

$$
\left(E_{f b, r e d o x}=e U\right)=\frac{1}{e k_{e t}[A] S R_{c t}}
$$

Equation 2

Where $e$ is the elementary charge, $k_{e t}$, is the charge-transfer constant in $\mathrm{cm}^{4} \mathrm{~s}^{-1},[\mathrm{~A}]$ is the concentration in the electrolyte of redox (donor/acceptor) pairs in $\mathrm{cm}^{-3}, S$ is the electrode area in $\mathrm{cm}^{2}$ and $R_{c t}$ is the charge-transfer resistance in $\Omega$. This is a very simple method for determining the DOS and, from it, the location of the VB and $C B$ edges, only requiring a potentiostat and a frequency response analyser. However, band positions are not determined as accurately as with other available techniques, because electrode polarization slightly changes the state distribution in the electrode. The band edge is usually determined as the energy at which the DOS is half the maximum value of the DOS within the band. However, this requires to reach potentials positive (VB) or negative $(C B)$ enough to reach that maximum, which in the case of PPy and aqueous solutions involves reaching the limits of the potential windows. Solvents with a wider potential window, such as acetonitrile, can be used, but this would prevent comparison with a potential scale based on the $\mathrm{H}^{+} / \mathrm{H}_{2}$ scale, relevant, e.g., for photocatalytic applications of PPy. In aqueous electrolytes, only the region corresponding to the edge of the PPy VB can be analysed (Fig. 2b). In addition, due to undesirable side reactions occurring above $1.0 \mathrm{~V} \mathrm{RHE}$, we could not extend our impedance-based analysis beyond this value. This prevented us from visualizing a range of DOS within the VB large enough to reach a maximum. For this reason, we determined the VB edge by extrapolating a fit to the region of linear increase of the DOS and finding the intercept of the best linear fit with the horizontal axis. Figure $2 b$ shows the DOS spectrum of our PPy films, from which a location of the VB edge at $0.95 \mathrm{~V}$ could be estimated. This value will be compared below with that obtained from IB-EC-STS.
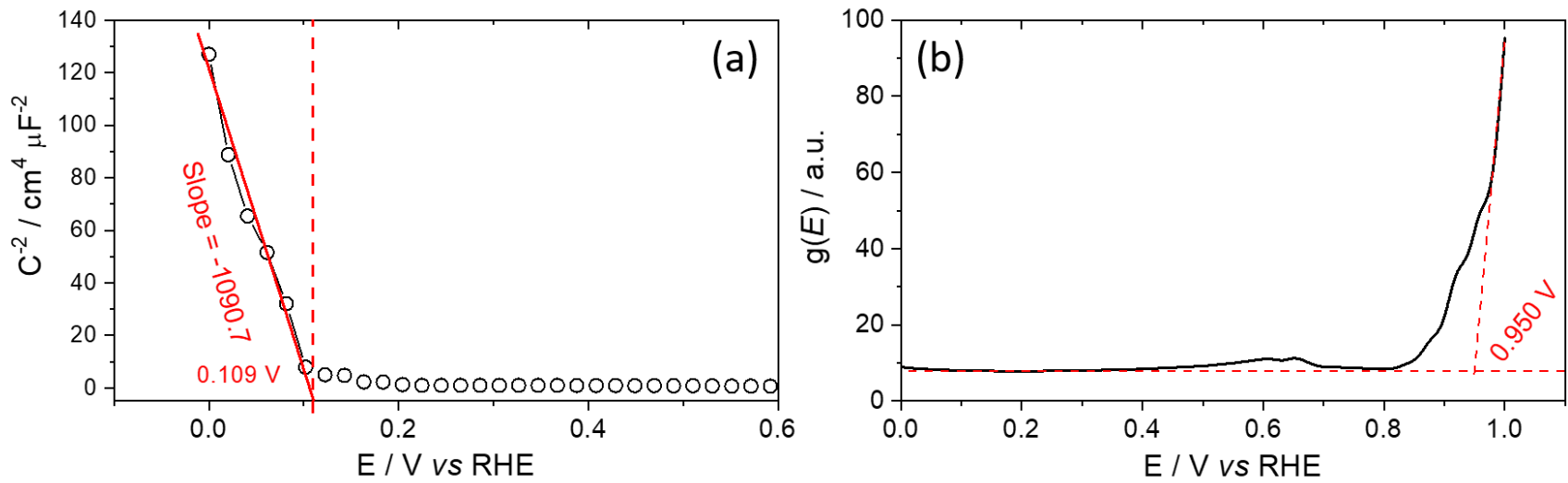

Figure 2. Mott-Schottky plot (a) and density of states (DOS) spectrum (b) of a thin film of polypyrrole grown on polycrystalline Pt after 20 voltammetric cycles.

\section{Image-based spectroscopy \\ electrochemical \\ scanning \\ tunnelling}

Fig. 3 shows an STM image recorded at a sample potential $E_{\text {Sample }}=0.25 \mathrm{~V}$, at which, according to the cyclic voltammogram in Fig. 1(b), the PPy film must have been slightly oxidised (i.e., some hole polarons must have been created), and an initial tip potential $E_{\text {Tip }}=0.55 \mathrm{~V}$. Under these conditions, imaging should be impossible, because electrons should tunnel from the sample (more negative potential, equivalently, higher $E_{\mathrm{F}}$ ) to the tip (more positive potential, equivalently, lower $E_{\mathrm{F}}$ ) but, according to the DOS spectrum in Fig. $2 \mathrm{~b}$, the $E_{\mathrm{F}}$ of the tip lies exactly within the PPy's band gap and there are no occupied states from which this could happen.

A clear image can however be obtained (Fig. 3a), which can only be explained if a half-filled polaron level is located at an energy higher than the tip's $E_{\mathrm{F}}$. In order to test this hypothesis, the tip potential was slowly scanned in the negative direction, which results in the image disappearing at $0.46 \mathrm{~V}$ (Fig. 3a). The image reappears if the tip potential is then stepped back to 0.55 $\mathrm{V}$, as shown in the lower part of the image (Fig. 3a). This allows us to locate the position of the hole polaron at $0.46 \mathrm{~V}$ vs. RHE, which is in good agreement with the small DOS between 0.4 and $0.7 \mathrm{~V}$ in the DOS spectrum in Fig. 2b. The behaviour observed in Fig. 3 can be explained as follows: when $E_{\text {tip }} \leq 0.46 \mathrm{~V}$ but more positive than the sample potential $(0.25 \mathrm{~V})$, the flow of electrons from the sample to the tip (as dictated by the positive tip bias) is not possible because the VB edge is lower in energy than the tip's Fermi level, and the electronic states in the CB are all empty at room temperature (Fig. 3c). On the contrary, when $E_{\text {tip }}>0.46 \mathrm{~V}$, the tip's energy level lies at a lower level than the half-filled polaron hole, which allows electrons to tunnel from the latter into the tip and a STM image to be established (Fig. 3b). 
(a)

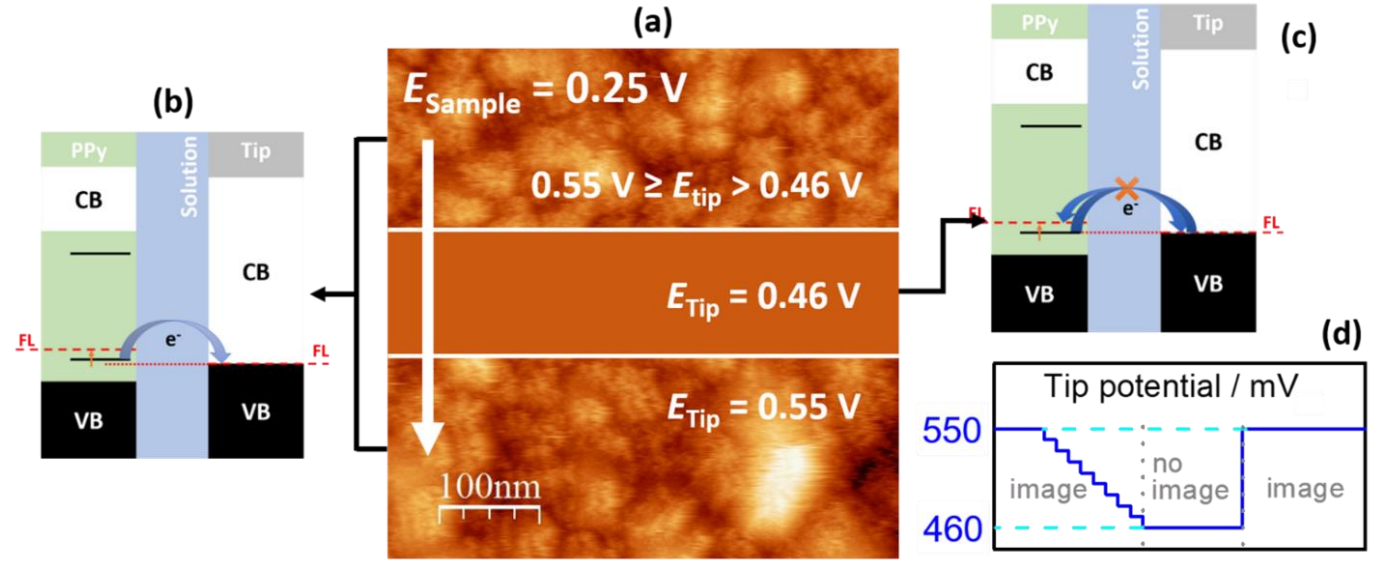

Figure 3. (a) STM image $\left(500 \times 500 \mathrm{~nm}^{2}\right)$ of a PPy film electrochemically grown on a Pt(111) electrode in $0.1 \mathrm{~mol} \mathrm{~L}^{-1} \mathrm{HClO}_{4}$ at $E_{\text {sample }}=0.25 \mathrm{~V}$. The scan direction is from top to bottom, as indicated by the white arrow. Initially, $E_{\text {tip }}=0.55 \mathrm{~V}$. $E_{\text {tip }}$ was then progressively made more negative in $0.01 \mathrm{~V}$ steps. The central region of the image was recorded at $E_{\text {tip }}=0.46 \mathrm{~V}$, and then $E_{\text {tip }}$ was stepped back to the initial value of $0.55 \mathrm{~V}$ before recording the bottom part of the image; (b) Schematic representation of the location of the different energy levels when $E_{\text {tip }}=0.55 \mathrm{~V}$, with electrons tunnelling from the intra-gap, half-filled, polaron hole state into the tip's Fermi level. The effect of band bending has been omitted for the sake of clarity and simplicity; (c) Schematic representation of the location of the different energy levels when $E_{\text {tip }} \leq 0.46 \mathrm{~V}$. Tunnelling is not possible either from the tip to the sample due to the positive tip bias, or from the sample to the tip, because there are no filled or partially filled electronic states above the tip's Fermi level. The effect of band bending has been omitted for the sake of clarity and simplicity; (d) Scheme illustrating the potential programme applied to the tip. $\digamma_{\mathrm{T}}=2 \mathrm{nA}$.

The experiment shown in Fig. 4 confirms that imaging in the top and bottom sections of Fig. 3a was due to tunnelling from intra-gap, half-filled polaron states. As in Fig. 3, $E_{\text {Tip }}$ and $E_{\text {Sample }}$ were initially set at 0.55 and $0.25 \mathrm{~V}$, respectively. A clear image was obtained under these conditions, reproducing the results in Fig. 3a. Instead of changing $E_{\text {Tip, }}$ as in Fig. 3, in this experiment $E_{\text {Tip }}$ was kept constant and $E_{\text {Sample }}$ was stepped to $0.10 \mathrm{~V}$ after having scanned approximately $1 / 4$ of the image area (Fig. 4a). According to the cyclic voltammogram in Fig. $1 \mathrm{~b}$, at $0.1 \mathrm{~V}$ the oxidised polymer units should be reduced, in other words, this potential step should result in the de-doping of PPy and the disappearance of the half-filled polaron states. As expected, the image vanishes after the potential step (Fig. 4a), confirming that imaging was due to tunnelling from the hole polaron states (see Figs. $4 \mathrm{~b}$ and $\mathrm{c}$ ). $E_{\text {Tip }}$ was then slowly made more positive until the image reappeared at $E_{\text {tip }}=0.95 \mathrm{~V}$ (Fig. 4a). This must correspond to the potential at which the Fermi level of the tip crosses the VB edge, allowing the tip to receive electrons from the VB (Figure 4d). This is in excellent agreement with the location of the VB edge as obtained from the EIS-derived DOS spectrum.

(a)

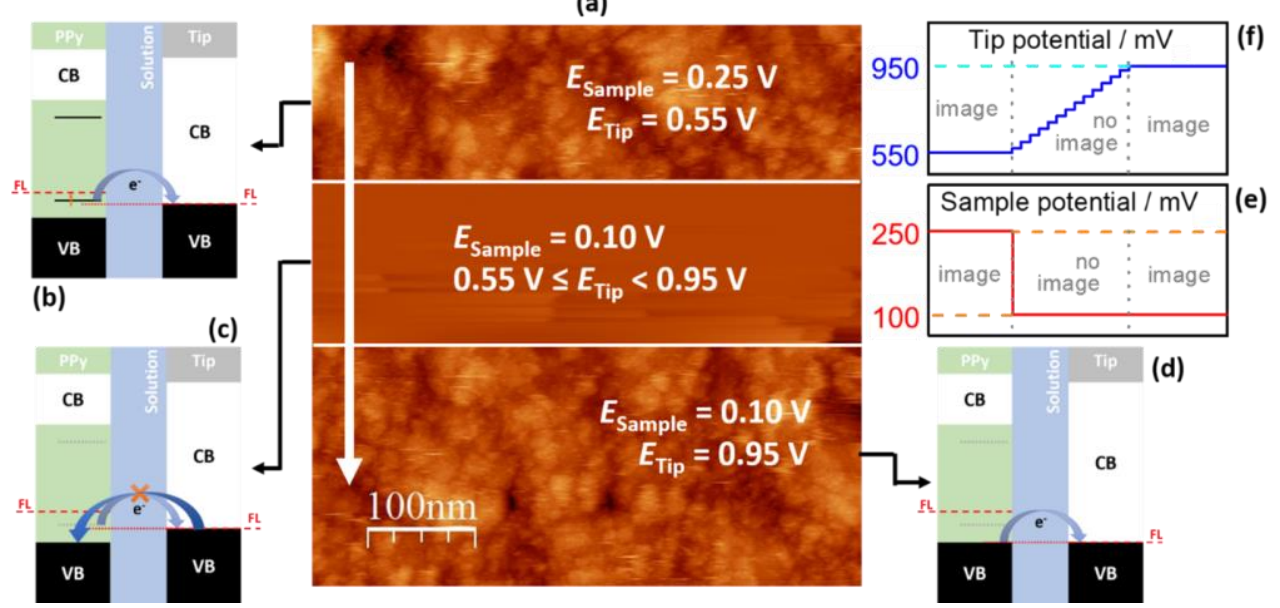

Figure 4. (a) STM image $\left(500 \times 500 \mathrm{~nm}^{2}\right)$ of a PPy film electrochemically grown on a $\mathrm{Pt}(111)$ electrode in $0.1 \mathrm{~mol} \mathrm{~L}^{-1} \mathrm{HClO}_{4}$ at initial $E_{\text {sample }}=0.25 \mathrm{~V}$ and $E_{\text {tip }}=$ $0.55 \mathrm{~V}$. The scan direction is from top to bottom, as indicated by the white arrow. $E_{\text {sample }}$ was stepped to $0.10 \mathrm{~V}$ approximately one quarter down the image. The central region of the image was recorded at $E_{\text {sample }}=0.10 \mathrm{~V}$ while $E_{\text {tip }}$ was increased from 0.55 to $0.95 \mathrm{~V}$ in $0.01 \mathrm{~V}$ steps. The bottom section of the image was recorded at $E_{\text {sample }}=0.10 \mathrm{~V}$ and $E_{\text {tip }}=0.95 \mathrm{~V}$; (b) Schematic representation of the location of the different energy levels when $E_{\text {tip }}=0.55 \mathrm{~V}$ and $E_{\text {sample }}=0.25 \mathrm{~V}$, with electrons tunnelling from the intra-gap, half-filled, polaron hole state into the tip's Fermi level. The effect of band bending has been omitted for the sake of clarity and simplicity; (c) Schematic representation of the location of the different energy levels when $E_{\text {sample }}=0.10 \mathrm{~V}$ and $0.55 \mathrm{~V} \leq E_{\text {tip }}<0.95 \mathrm{~V}$. Tunnelling is not possible either from the tip to the sample due to the positive tip bias or from the sample to the tip, because there are no filled or partially filled electronic states above the tip's Fermi level. The effect of band bending has been omitted for the sake of clarity and simplicity; (d) Schematic representation of the location of the different energy levels when $E_{\text {sample }}=0.10 \mathrm{~V}$ and $E_{\text {tip }}=0.95 \mathrm{~V}$. Tunnelling is now possible from PPy's VB into the tip's Fermi level; (e) and (f) Schemes illustrating the potential programmes applied to sample and tip, respectively. $l_{\mathrm{T}}=2 \mathrm{nA}$. 
Because hole polaron levels are half filled, tunnelling from the tip to these levels must be possible under negative tip bias. This is confirmed by the results in Fig. 5 . In this case, $E_{\text {Sample }}$ was set at $0.25 \mathrm{~V}$ and $E_{\text {Tip }}$ was initially set at $0.49 \mathrm{~V}$. As we have seen above, polaron states have already been created at this

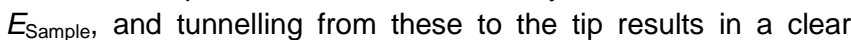
STM image (Figs. 5a and b). $E_{\text {Tip }}$ was then slowly scanned in the negative direction in $0.10 \mathrm{~V}$ steps, until the polaron level is crossed at $0.43 \mathrm{~V}$ (please note that a Pt wire was used as quasireference in these spectra, which accounts for the small difference in the location of the polaron level in Figs. 3 and 5), at which the image disappears for the reasons explained above (Figs. $5 \mathrm{a}$ and $\mathrm{c}$ ). However, when the $E_{\text {Tip }}$ reaches a value of $0.29 \mathrm{~V}$ and the tip crosses the sample's Fermi level, the STM image is recovered because now electrons can tunnel from the tip to the half-filled polaron level (Figs. 5a and d).

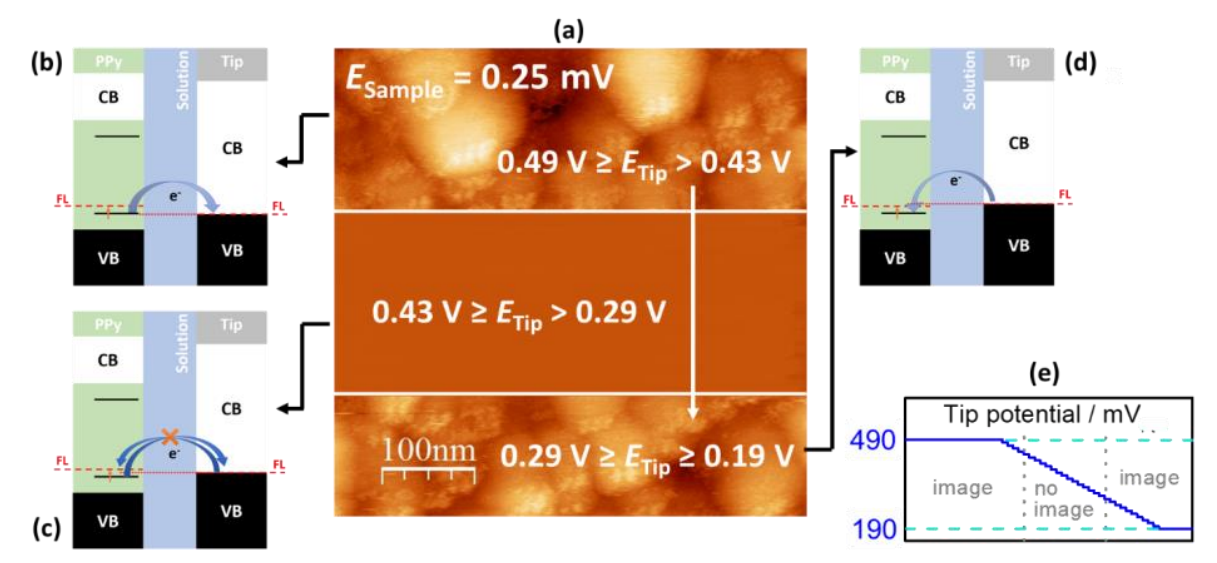

Figure 5. (a) STM image $\left(500 \times 500 \mathrm{~nm}^{2}\right)$ of a PPy film electrochemically grown on a Pt(111) electrode in $0.1 \mathrm{~mol} \mathrm{~L}^{-1} \mathrm{HClO}_{4}$ at $E_{\text {sample }}=0.25 \mathrm{~V}$. The scan direction is from top to bottom, as indicated by the white arrow. Initially, $E_{\text {tip }}=0.49 \mathrm{~V}$, and was then progressively made more negative in $0.01 \mathrm{~V}$ steps. The central region of the image was recorded at $E_{\text {tip }}$ between 0.43 and $0.29 \mathrm{~V}$, and the bottom section of the image at $E_{\text {tip }}$ between 0.29 and $0.19 \mathrm{~V}$; (b) Schematic representation of the location of the different energy levels when $E_{\text {tip }}>0.43 \mathrm{~V}$, with electrons tunnelling from the intra-gap, half-filled, polaron hole state into the tip's Fermi level. The effect of band bending has been omitted for the sake of clarity and simplicity; (c) Schematic representation of the location of the different energy levels when $E_{\text {tip }}$ is between 0.43 and $0.29 \mathrm{~V}$. Tunnelling is not possible either from the tip to the sample, due to the positive tip bias, or from the sample to the tip, because there are no filled or partially filled electronic states above the tip's Fermi level; (d) Schematic representation of the location of the different energy levels when $E_{\text {tip }}$ between 0.29 and $0.19 \mathrm{~V}$. Tunnelling is now possible from the tip to the sample due to the negative tip bias and the presence of partially filled polaron levels below the tip's Fermi level. The effect of band bending has been omitted for the sake of clarity and simplicity; (e) Scheme illustrating the potential programme applied to the tip. $l_{T}$ $=2 \mathrm{nA}$. In schematics b, c, d the effect of band bending has been omitted for the sake of clarity and simplicity.

Our IB-EC-STS results allow us to build a quantitative energy diagram for PPy (Fig. 6). For the absolute potential scale a value of $-4.44 \mathrm{eV}$ for the absolute potential of the SHE was used, as recommended by Trasatti ${ }^{[31]}$, which sets the absolute potential of the RHE at $\mathrm{pH} 1$ at $-4.38 \mathrm{eV}$. The diagram is consistent with others reported in the literature obtained by spectroscopic methods $^{[1,15,32]}$.

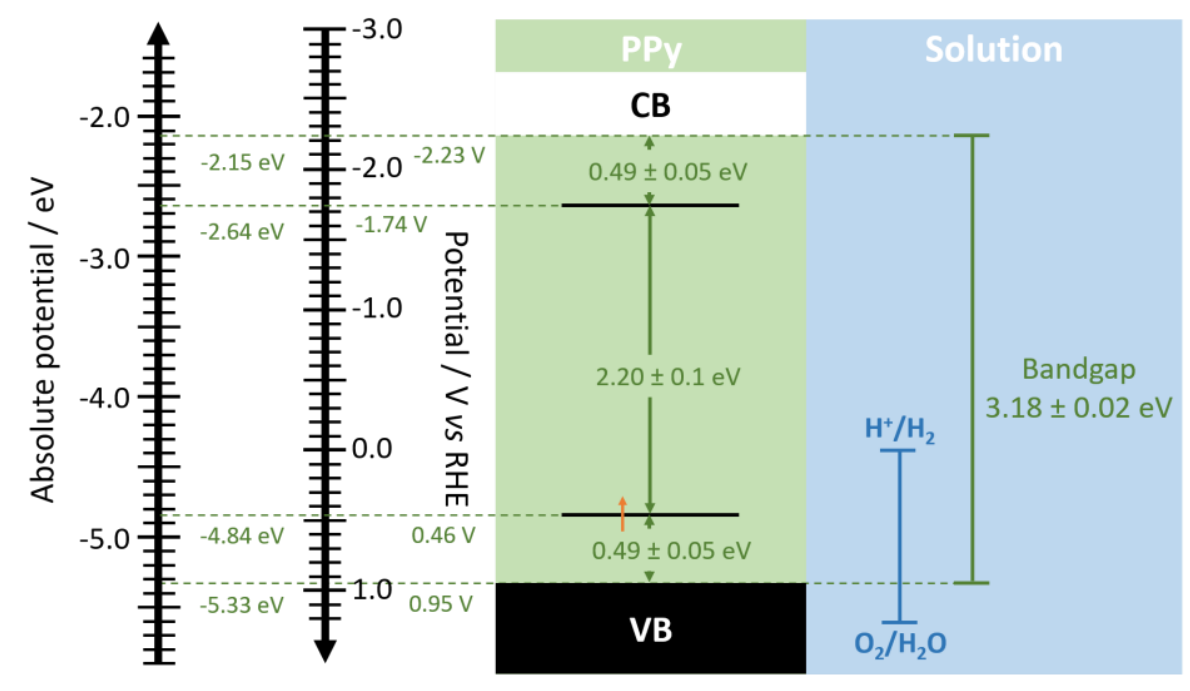

Figure 6. Band diagrams of a thin polypyrrole film on $\mathrm{Pt}$ as deduced from image-based electrochemical scanning tunnelling spectroscopy in 0.1 mol $\mathrm{L}^{-1} \mathrm{HCIO}_{4}$. The energy levels are indicated in both the RHE and the absolute potential scales. 
We would like to finish with a brief comment regarding the value of the flat-band potential as obtained from a Mott-Schottky analysis of the interfacial capacitance. The applicability and pitfalls of Mott-Schottky analysis and other methods for the determination of flat-band potentials have recently been discussed by Hankin et al. ${ }^{[33]}$ As discussed by them, Mott Schottky analysis rests on the assumption that the capacitance of the interface is dominated by that of the semiconductor's space-charge region or that, equivalently, the potential drop across the electrolyte side of the interface is much smaller than that across the space-charge region, which might not always be the case and would limit the applicability of the Mott-Schottky equation. We would like to add here that, for materials like PPy for which the doping level is continuously increasing with increasing potential (other materials like hydrogen tungsten bronzes, whose conductivity depends on the $\mathrm{W}(\mathrm{V}) / \mathrm{W}(\mathrm{VI})$ ratio, could also fall in this category), the flat-band potential must also be continuously changing, which will further limit Mott-Schottky's applicability. In our particular example, we would expect the flatband potential of undoped PPy to lie close, if not exactly at, the centre of the gap, i.e., around $-0.64 \mathrm{~V}$ vs. RHE. On the contrary, Mott-Schottky delivers a much more positive value of the apparent flat-band potential of ca. $0.11 \mathrm{~V}$ vs. RHE, only $0.35 \mathrm{eV}$ higher than the polaron level. The location of the flat band potential will be dragged down in energy as partially filled states are created some hundreds of $\mathrm{mV}$ above the band edge, and the apparent flat-band potential might therefore correspond instead to that of doped PPy, but the question is then what level of doping leads to this value. Alternatively, the apparent flatband potential might indicate the onset potential of PPy oxidation (i.e., of PPy doping) which would agree with the cyclic voltammogram (Fig. 1b) and the observation in Fig. 4 that tunnelling from the sample to the tip is not possible when $E_{\text {sample }}$ $\leq 0.1 \mathrm{~V}$ and $E_{\text {tip }}$ is more positive than $E_{\text {sample }}$ but more negative than $0.95 \mathrm{~V}$ (the valence band edge). More research is probably needed to solve this question.

\section{Conclusion}

IB-EC-STS was demonstrated as a tool that allows to achieve a complete characterization of the band structure of a PPy film on Pt, which was consistent with the DOS spectrum obtained from electrochemical impedance spectroscopy. The advantage of IB-EC-STS over other methods is that the information regarding the electronic levels is obtained directly in a physically meaningful scale and does not rely on the application of a model. It also allows the direct observation of the emergence/vanishing of intragap polaron states associated to the doping/de-doping process.

\section{Acknowledgements}

The support of FAPESP (2011/10897-2, 2013/07296-2), CsF-PVE (99999.007708/2015-07), CAPES (PVE Project No A090/2013; Finance Code 001) and CNPq is gratefully acknowledged.

Keywords: polypyrrole $\cdot$ electronic structure $\cdot$ electrochemical scanning tunnelling microscopy $\bullet$ impedance spectroscopy

\section{References}

[1] W. H. Smyrl, M. Lien, in Appl. Electroact. Polym., Springer Netherlands, Dordrecht, 1993, pp. 29-74.

[2] R. J. Waltman, J. Bargon, P. Chemie, U. Bonn, W. Germany, R. J. Waltman, J. Bargon, Can. J. Chem. 1986, 64, 76-95.

[3] P. Y. Yu, M. Cardona, in Fundam. Semicond., 2010, pp. 17-106.

[4] P. Y. Yu, M. Cardona, in Fundam. Semicond., 2010, pp. 203-241.

[5] J. L. Bredas, G. B. Street, Acc. Chem. Res. 1985, 18, 309-315.

[6] A. R. Nagaraja, N. H. Perry, T. O. Mason, Y. Tang, M. Grayson, T. R. Paudel, S. Lany, A. Zunger, J. Am. Ceram. Soc. 2012, 95, 269-274.

[7] A. O. Patil, A. J. Heeger, F. Wudl, Chem. Rev. 1988, 88, 183-200.

[8] I. Sakellis, A. N. Papathanassiou, J. Grammatikakis, Appl. Phys. Lett. 2008, 92, 222108.

[9] G. Paasch, S. Scheinert, A. Petr, L. Dunsch, Russ. J. Electrochem. 2006, 42, 1161-1168.

[10] M. S. Sercheli, L. O. S. Bulho, 2000, 631, 631-634.

[11] H. E. Toma, Química Nov. na Esc. 1997, 8-12.

[12] R. Faez, C. Reis, P. FREITAS, O. Kosima, Química Nov. na Esc. 2000 , 13-18.

[13] L.-X. Wang, X.-G. Li, Y.-L. Yang, React. Funct. Polym. 2001, 47, 125139.

[14] Z.-B. Huang, G.-F. Yin, X.-M. Liao, J.-W. Gu, Front. Mater. Sci. 2014, 8, 39-45.

[15] E. M. Genies, G. Bidan, A. F. Diaz, J. Electroanal. Chem. 1983, 149 $101-113$.

[16] K. Gentz, K. Wandelt, Chim. Int. J. Chem. 2012, 66, 44-51.

[17] H. J. W. Zandvliet, A. van Houselt, Annu. Rev. Anal. Chem. 2009, 2 37-55.

[18] A. K. Yagati, J. Min, J.-W. Choi, in Mod. Electrochem. Methods Nano, Surf. Corros. Sci., InTech, 2014.

[19] S. Wakabayashi, H. Kato, M. Tomitori, O. Nishikawa, J. Appl. Phys. 1994, 76, 5595-5597.

[20] I. Díez-Pérez, P. Gorostiza, F. Sanz, J. Electrochem. Soc. 2003, 150, B348.

[21] G. Garcia-Belmonte, J. Bisquert, Electrochim. Acta 2002, 47, 42634272.

[22] J. Bisquert, G. G. Belmonte, F. F. Santiago, N. S. Ferriols, M Yamashita, E. C. Pereira, Electrochem. commun. 2000, 2, 601-605.

[23] T. F. Otero, Polym. Rev. 2013, 53, 311-351.

[24] G. Paasch, Synth. Met. 2001, 119, 233-234.

[25] G. Paasch, Electrochim. Acta 2002, 47, 2049-2053.

[26] J. Bisquert, A. Compte, J. Electroanal. Chem. 2001, 499, 112-120.

[27] R. Gonçalves, A. A. A. Correa, R. Pereira, E. C. C. Pereira, Electrochim. Acta 2016, 190, 329-336.

[28] A. A. Correa, R. Gonçalves, R. Pereira, E. C. Pereira, J. Appl. Polym. Sci. 2017, 134, DOI 10.1002/app.44368.

[29] K. Gmucová, V. Nádaždy, F. Schauer, M. Kaiser, E. Majková, J. Phys Chem. C 2015, 119, 15926-15934

[30] V. Nádaždy, F. Schauer, K. Gmucová, Appl. Phys. Lett. 2014, 105 142109.

[31] S. Trasatti, J. Electroanal. Chem. Interfacial Electrochem. 1986, 209 417-428.

[32] M. J. L. J. L. Santos, A. G. G. Brolo, E. M. M. Girotto, Electrochim. Acta 2007, 52, 6141-6145.

[33] A. Hankin, F. E. Bedoya-Lora, J. C. Alexander, A. Regoutz, G. H Kelsall, J. Mater. Chem. A 2019, 7, 26162-26176. 


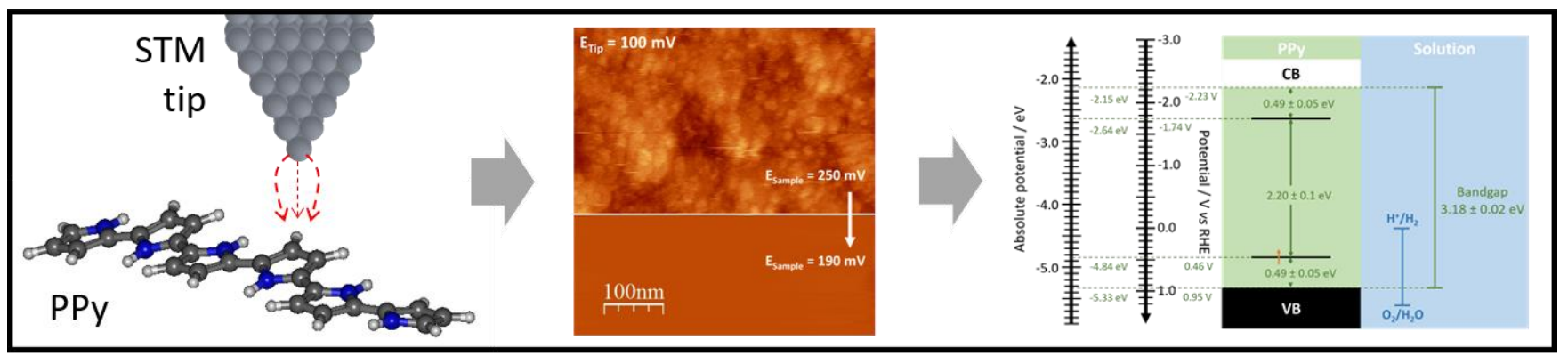

The difficulties associated to the use of scanning tunneling spectroscopy (STS) in electrochemical environments can be circumvented by using imaged-based electrochemical scanning tunneling spectroscopy (IB-EC-STS). Application of IB-EC-STS to a polypyrrole film allows to determine the edge of the valence band the intragap polaron states created upon partial oxidation ( $p$-doping) of the polymer in the absolute and hydrogen potential scales.

Institute and/or researcher Twitter usernames: Angel C Ciscar (@c_ciscar) 